\title{
Risk Assessment in Complex Interacting Infrastructure Systems
}

$\begin{array}{ccc}\text { D. E. Newman } & \text { Bertrand Nkei } & \text { B. A. Carreras } \\ \text { Physics } & \text { Oak Ridge } & \text { Oak Ridge } \\ \text { Department } & \text { National } & \text { National } \\ \text { University of } & \text { Laboratory, } & \text { Laboratory, } \\ \text { Alaska, } & \text { Oak Ridge,TN } & \text { Oak Ridge,TN } \\ \text { Fairbanks, AK } & \text { 37831USA } & \text { 37831USA } \\ \text { 99775USA } & \text { nnb@fed.ornl.g } & \text { carrerasba@or } \\ \text { ffden@uaf.edu } & \text { ov } & \text { nl.gov }\end{array}$

\begin{abstract}
Critical infrastructures have some of the characteristic properties of complex systems. They exhibit infrequent large failures events. These events, though infrequent, often obey a power law distribution in their probability versus size. This power law behavior suggests that ordinary risk analysis might not apply to these systems. It is thought that some of this behavior comes from different parts of the systems interacting with each other both in space and time. While these complex infrastructure systems can exhibit these characteristics on their own, in reality these individual infrastructure systems interact with each other in even more complex ways. This interaction can lead to increased or decreased risk of failure in the individual systems. To investigate this and to formulate appropriate risk assessment tools for such systems, a set of models are used to study to impact of coupling complex systems. A probabilistic model and a dynamical model that have been used to study blackout dynamics in the power transmission grid are used as paradigms. In this paper, we investigate changes in the risk models based on the power law event probability distributions, when complex systems are coupled.
\end{abstract}

\section{Introduction}

It is fairly clear that many important infrastructure systems exhibit the type of behavior that has come to be associated with "Complex System" dynamics. These systems range from electric power transmission and distribution systems, through communication networks, commodity transportation infrastructure arguably all the way to the economic markets themselves. There has been extensive work in the modeling of some of these different systems. However, because of the intrinsic complexities involved, modeling of the interaction between these systems has been limited [1,2]. While understandable from the standard point of view that espouses understanding the components of a large complex system before one tries to understand the entire system, this approach can unfortunately overlook important consequences

\author{
I. Dobson \\ ECE \\ Department, \\ University of \\ Wisconsin, \\ Madison, WI \\ 53706 USA \\ dobson@engr. \\ wisc.edu
}

\author{
V. E. Lynch \\ Oak Ridge \\ National \\ Laboratory, \\ Oak Ridge, $T N$ \\ 37831 USA \\ lynchve@ornl.g \\ ov
}

\author{
Paul Gradney \\ Physics \\ Department \\ University of \\ Alaska, \\ Fairbanks, $A K$ \\ 99775 USA
}

of the coupling of these systems that impact their safe operation and overlooks critical vulnerabilities of these systems. At the same time, one cannot simply take the logical view that the larger coupled system is just a new larger complex system because of the heterogeneity introduced through the coupling of the systems. While the individual systems may have a relatively homogeneous structure, the coupling between the systems is often both in terms of spatial uniformity and in terms of coupling strength, fundamentally different (Figure 1). This in the most extreme case leads to uncoupled systems but in the more normal region of parameter space in which the intersystem coupling is weaker or topologically different then the intra-system coupling can lead to important new behavior. Understanding the effect of this coupling on the system dynamics is necessary if we are to accurately develop risk models for the different infrastructure systems individually or collectively.

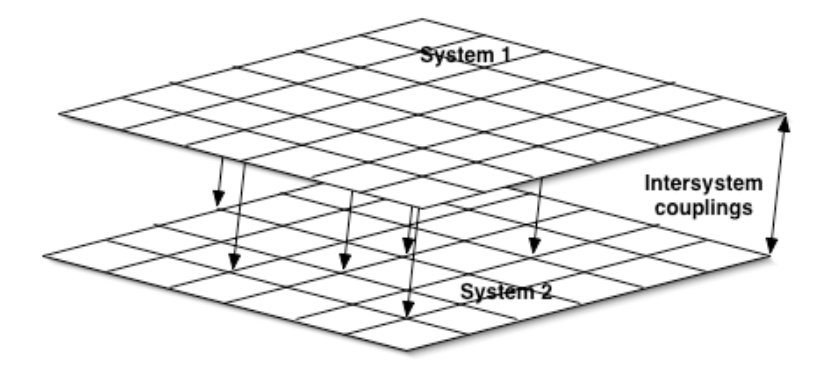

Figure 1: Cartoon of two homogeneous systems with
a heterogeneous coupling

Examples of the types of potential coupled infrastructure systems to which this would be relevant include powercommunication systems, power-market systems, communication-transportation systems, and even marketmarket systems. Interesting examples of these interactions are discussed in ref. [3]. The effect of this coupling can be critical and obvious for systems that are strongly coupled such as the power - market coupled system. Perturbations in one can have a rapid and very visible impact on the other. In fact, in many ways such systems are often thought of as one larger system even though the coupling is not homogeneous and each of the component systems (namely the market and the power 
transmission system) can have their own separate perturbations and dynamics. For other less tightly coupled systems, such as power-communications systems, the effect can be much more subtle but still very important. In such systems small perturbations in one might have very little obvious effect on the other system, yet the effect of the coupling of the two systems can have a profound effect on the risk of large, rare disturbances.

In this paper, we will investigate some of these effects using two different approaches. First we will use a simple probabilistic model for cascading failures (CASCADE) that has been extensively studied for individual systems [4-6]. This model allows us to probe the impact of the coupling on the failure risks and the critical point that has been previously found for the uncoupled systems. This model also has the advantage of allowing some analytic solutions. Next we will present results from a dynamical model of coupled complex systems. This model has dynamic evolution and many of the characteristics found in complex systems.

Throughout this paper for reference purposes we will use the power transmission system as the primary system and the communications systems as the coupled secondary system. In reality, the models discussed have very little specific to these systems. They will be used so the results are more general in nature and we use these reference systems simply to be able to give concrete examples of the actions and effects we discuss.

Many complex systems are seen to exhibit similar characteristics in their failures. While it is useful and important to do a detailed analysis of the specific causes of these failures such as individual blackouts, it is also important to understand the global dynamics of the systems like the power transmission network. This allows some insight into the frequency distribution of these events (e.g. blackouts) that the system dynamics creates. There is evidence that global dynamics of complex systems is largely independent of the details of the individual triggers such as shorts, lightning strikes etc in power systems. In this paper, we focus on the intrinsic dynamics of failures and how this complex system dynamics impacts failure risk assessment in interconnected complex systems. It is found, perhaps counter intuitively, that even weak coupling of complex systems can have adverse effects on both systems and therefore risk analysis of an isolated system must be approached with care.

Several particular issues induced by the interdependence of systems will be addressed in this paper. The first one is how coupling between the systems modifies conditions for safe operation. These systems are characterized by a critical loading $[7,8]$. They must operate well-below this critical loading to avoid "normal accidents" [9] and large scale failures. We will explore how the coupling between systems changes the value of this critical loading.

We will also consider the effect of the heterogeneity introduced through in two different ways. Through the different properties of each individual system, like having different critical points, and the coupling of the systems.

Finally we will contrast probabilistic models with dynamical models in order to see the effect of memory in the system impacts the consequences of the couplings.

The rest of the paper will be organized as follows: Section 2 reviews some of the characteristics of complex systems. Section 3 contains a description of the coupled cascade model and results from that model. Section 4 describes the dynamic model with results from that model, followed by section 5 that has a discussion of the implications of these results and conclusions.

\section{Coupled CASCADE model}

\subsection{Individual CASCADE model}

The basic CASCADE model [4-6] has $n$ identical components with random initial loads. For each component the minimum initial load is $L_{\min }$ and the maximum initial load is $L_{\max }$. For $j=1,2, \ldots, n$, component $j$ has an initial load of $l_{j}$ that is a random variable uniformly distributed in $\left[L_{\min }, L_{\max }\right] . l_{1}, l_{2}, \cdots$, $l_{n}$ are independent. Components fail when their load exceeds $L_{\text {fail }}$. When a component fails, a fixed amount of load $p$ is transferred to each of the components.

To start the cascade, we assume an initial disturbance that loads each component with an additional amount, $d$. Components may then fail depending on their initial loads, $l_{j}$, and the failure of any of these components will distribute an additional load, $p \geq 0$, that can cause further failures in a cascade. This model describes the cascading failure as an iterative process. In each iteration, loads fail as the transfer load, $p$, from other failures makes them reach the failure limit. The process stops when none of the remaining loads reaches the failure limit. It is useful to define $\lambda \equiv n p$, the total load transferred from a failing component. This system is found to have a transition in the probability of system wide failures $\left(P_{\infty}\right)$ at a critical value of $\lambda$. As shown in Fig. 2 , when $\lambda<\lambda_{c}$, where $\lambda_{c}$ is the critical value of $\lambda, P_{\infty}=0$. However, above the critical value for $\lambda$, system wide failures are possible. In the CASCADE model if we assume a uniform random distribution of loads, the critical point is $\lambda_{\mathrm{c}}=1$.

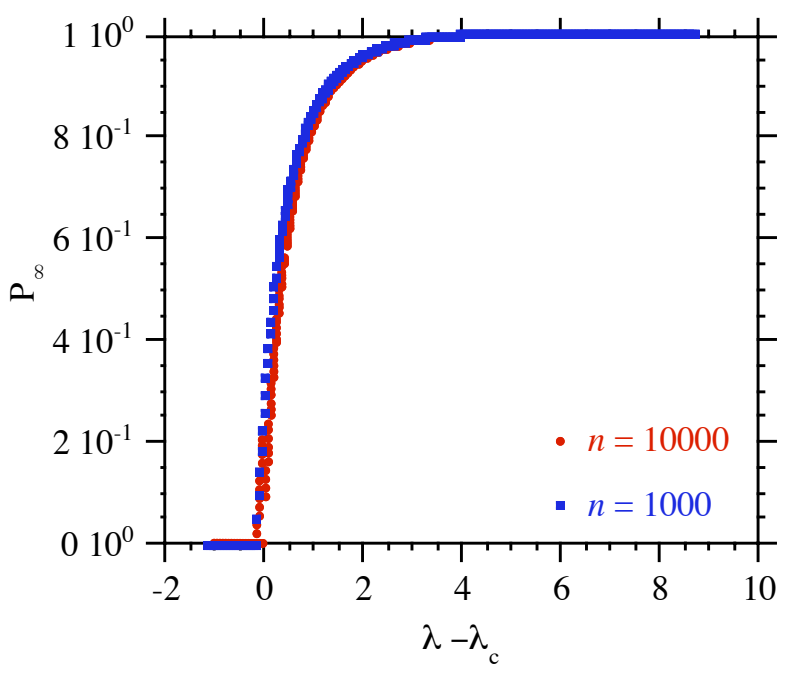
Figure 2: Probability of cascade events of the system
size as a function of $\lambda$

An important characteristic of the CASCADE model is that around the critical point, the probability distribution 
function (pdf) of the size of the failures develops a power law tail. In the uniform load case, this power law tail has a characteristic exponent of approximately -1.5 . This power law behavior is important because the effect of a failure is proportional to its size so if the probability of failures falls as a power law less steep then -2.0 , the large failures dominate the "cost" of failure.

\subsection{Coupled CASCADE models}

Generalizing the CASCADE model to a pair of coupled CASCADE systems is straightforward. We consider two systems $L$ and $M$ with random loads (normalized on 0 to 1 ):

$$
\begin{array}{ll}
\text { System } L & l_{i} \in[0,1] i=1, \ldots n_{L} \\
\text { System } M & m_{j} \in[0,1] j=1, \ldots n_{M}
\end{array}
$$

At the beginning of each "day" (realization), the random initial loads are generated. We will simplify the situation by considering only initial perturbations in the system $L$. As an initial perturbation, we add an increment $d$ to all loads of the components in system $L$. As before, a component fails if its normalized load is greater than 1. For each failed component, we transfer a load $p_{\mathrm{LL}}$ to the loads of all other components in the same way that we did in the individual model. Now however, when component i of $L$ fails, all loads of the components of system $M$ are increased by an amount $\mathrm{p}_{\mathrm{ML}}$. This cross system loading is the inter-system coupling. It should not be thought of as actually distributing the load for $L$ to the other system, rather one can think of it as an increased stress in system $\mathrm{M}$ due to failures in system $\mathrm{L}$.

Likewise, when a component in the system $M$ fails a load $\mathrm{p}_{\mathrm{MM}}$ is transferred to all loads of the other components of the system $M$ in the same way as was done in system $L$. Finally, we have the back cross loading coming when a component $\mathrm{j}$ of $M$ fails then all loads of the components of system $L$ are increased by an amount $\mathrm{p}_{\mathrm{LM}}$.

The basic steps of the algorithm proceed as follows:

At Step $t$

1) Test stability of all loads in $L$ based on their values at step $t-1$.

2) Test possible transfer from $L$ to $M$ based on the load values at step $t-1$.

3) Test stability of all loads in $M$ based on their values at step $t-1$.

4) Test possible transfer from $M$ to $L$ based on the load values at step $t$-1.

Now update all loads

At the end of each "day" we collect information on how many components failed in $L$ and how many in $M$, how long the whole cascade took, and accumulate information for a pdf of failures in both systems. We also accumulate data per iteration from each system, in order to calculate the number of failures per iteration.

The CASCADE model can be re-interpreted as a branching process [10]. This allows the application of the branching process methods [11] to analyze and interpret the results of the cascade model. In trying to understand the consequences of the coupled CASCADES model, we approximate it by a branching process. For simplicity we assume that the two systems have the same size and have symmetric couplings. From the load transfers we can construct the corresponding the transition probability as was done in Ref.[10]. In this case, we define $\lambda_{i j}=n p_{i j}$. Then if $F_{L}(t)$ and $\mathrm{F}_{\mathrm{M}}(\mathrm{t})$ are the mean number of failures in systems $L$ and $M$ respectively, we have

$$
\left(\begin{array}{l}
F_{L}(t) \\
F_{M}(t)
\end{array}\right)=\left(\begin{array}{ll}
\lambda_{L L} & \lambda_{L M} \\
\lambda_{M L} & \lambda_{M M}
\end{array}\right)\left(\begin{array}{l}
F_{L}(t-1) \\
F_{M}(t-1)
\end{array}\right)
$$

with

$$
\left(\begin{array}{l}
F_{L}(1) \\
F_{M}(1)
\end{array}\right)=\left(\begin{array}{l}
\theta \\
0
\end{array}\right)
$$

and

$$
\theta=n d
$$

This a 2 type branching process approximation to the evolution of the means in the coupled CASCADE model that generalizes the approximation in [10]. Therefore, iteration of Eq. (1) with the initial condition (2) leads to

$$
\left(\begin{array}{l}
F_{L}(t) \\
F_{M}(t)
\end{array}\right)=\left(\begin{array}{ll}
\lambda_{L L} & \lambda_{L M} \\
\lambda_{M L} & \lambda_{M M}
\end{array}\right)^{t-1}\left(\begin{array}{l}
\theta \\
0
\end{array}\right)
$$

To solve this system of equations we have to find the eigenvalues of the matrix, they are

$$
\lambda_{ \pm}=\frac{1}{2}\left[\lambda_{L L}+\lambda_{M M} \pm \sqrt{\left(\lambda_{L L}-\lambda_{M M}\right)^{2}+4 \lambda_{L M} \lambda_{M L}}\right]
$$

Since all $\lambda$ 's are positives the largest eigenvalue is $\lambda_{+}$. Because of the initial conditions,

$$
F_{L}(t)=\theta \frac{\left(\lambda_{+}-\lambda_{M M}\right) \lambda_{+}^{t-1}+\left(\lambda_{+}-\lambda_{L L}\right) \lambda_{-}^{t-1}}{\sqrt{\left(\lambda_{L L}-\lambda_{M M}\right)^{2}+4 \lambda_{L M} \lambda_{M L}}}
$$

and

$$
F_{M}(t)=\theta \frac{\lambda_{+}^{t-1}-\lambda_{-}^{t-1}}{\sqrt{\left(\lambda_{L L}-\lambda_{M M}\right)^{2}+4 \lambda_{L M} \lambda_{M L}}} \lambda_{M L}
$$

As an easy test to start comparing the code, we could use $\lambda_{L L}=\lambda_{M M}=\lambda$ and $\lambda_{L M}=\lambda_{M L}=\delta$. In this case, $\lambda_{ \pm}=\lambda \pm \delta$ and

$$
F_{L}(t)=\theta\left[\frac{(\lambda+\delta)^{t-1}+(\lambda-\delta)^{t-1}}{2}\right]
$$

and

$$
F_{M}(t)=\left[\frac{(\lambda+\delta)^{t-1}-(\lambda-\delta)^{t-1}}{2}\right]
$$


Because of the cascade nature of the process, the average number of failures diverges if the largest eigenvalue is greater than 1 and converges if it is less than 1 . Therefore the critical point is now given by

$$
\lambda_{c}=1-\delta
$$

This means that the coupling of the systems has shifted the critical point to a lower value of $\lambda$. The size of this shift is related to the strength of the coupling. This shift makes the system more susceptible to large failures. It is again important to note that the inter-system load transfer is intrinsically different then the intra-system load transfer. It is this difference that allows the shift in the critical point.

\subsection{Numerical results}

Numerically one can explore the parameter space to investigate the transition characteristics as a function of these parameters. Initially, we have considered only cases with $\lambda_{L L}=\lambda_{M M}=\lambda$ and $\lambda_{L M}=\lambda_{M L}=\delta$ in order to explore a small space to start with. For this situation we have only to worry about a single new parameter $\delta$. Calculations have been done for two systems of size 400 .

For a fixed initial perturbation, $\theta=0.2$, applied to the system $L$, we can see that the frequencies of the cascades in system $M$ increases with $\lambda+\delta$. This increase is faster when the system is close to the critical point (Fig. 3).

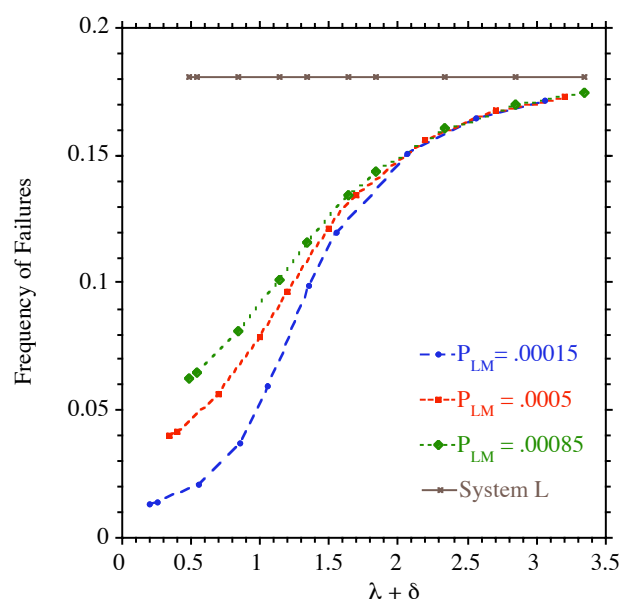

Figure 3: Frequency of failure as a function of $\lambda+\delta$

Because system $M$ is not perturbed, it is clear that the failures in system $L$ drive the failures in system $M$. Below the critical point, the effect is weak. However, at the critical point both systems become strongly coupled. They act more like a single system.

In addition to the drive of system $M$ by system $L$, there is clear feedback of system $M$ on system $L$, because the critical point is shifted downwards as given by Eq. (9). The numerical results are consistent with the analytical calculation: both systems have the same critical point and the critical point is given by the largest eigenvalue $\lambda+\delta$. This is shown in Figs. 4 and 5. . In Fig.4, we have plotted the probability of a system-size failure (the system as size 400) for system $L$ as a function of $\lambda$ for the different values of the coupling $p_{\mathrm{LM}}$. Here, $p_{\mathrm{LM}}$ is the load transferred to each load of the system $L$ by each failure in the system $M$. Then, $\delta=n p_{\mathrm{LM}}$. We can see that the critical point is shifted to lower $\lambda$ as $p_{\mathrm{LM}}$ increases. Note that with the strongest coupling there is almost a factor of 2 change in the critical point.

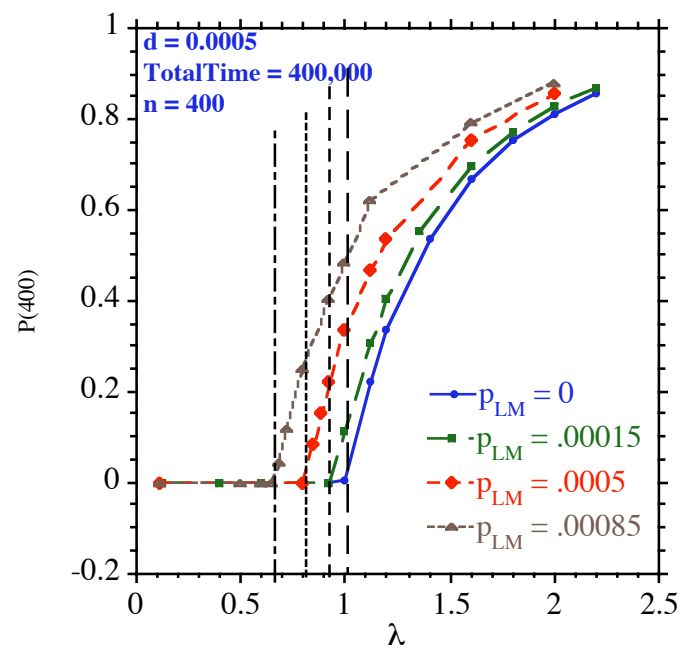

\section{Figure 4: Probability of cascade events of the system size as a function of $\lambda$}

That the shift in the critical point is given by $\delta$ is clearly shown in Fig. 5, where we have replotted the data in Fig. 4 as a function of $\lambda+\delta$. A universal curve emerges from this plot. Plots of the system-size failure probability for system $M$ are identical to the plots for system $L$.

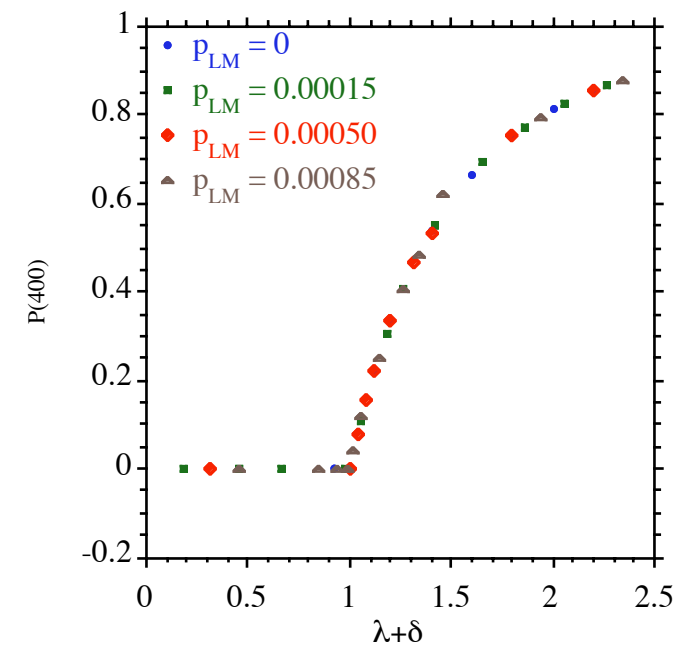

\section{Figure 5: Probability of system size cascade events as a function of $\lambda+\delta$}

In Fig. 6, we have plotted the pdf of the cascade size for $\lambda=0.95$ and $\delta=0.06$ (just 0.01 above the threshold). Keep in mind that for system $\mathrm{M}$ there would be no failures at all if the systems were uncoupled while for system $L$, without the coupling the system would still be significantly sub-critical. The pdf of failures for system $L$ has the usual slope of -1.5 . Remarkably, the slope for system $M$ is actually lower than for 
system $L$ and is close to -1.2 . The probability of small cascade in $L$ triggering cascades in $M$ is small. However, large cascades in $L$ often trigger cascades in $M$. Therefore, the probability of system wide cascades is practically the same in both systems. It is this combination that leads to the shallower slope for system $M$.

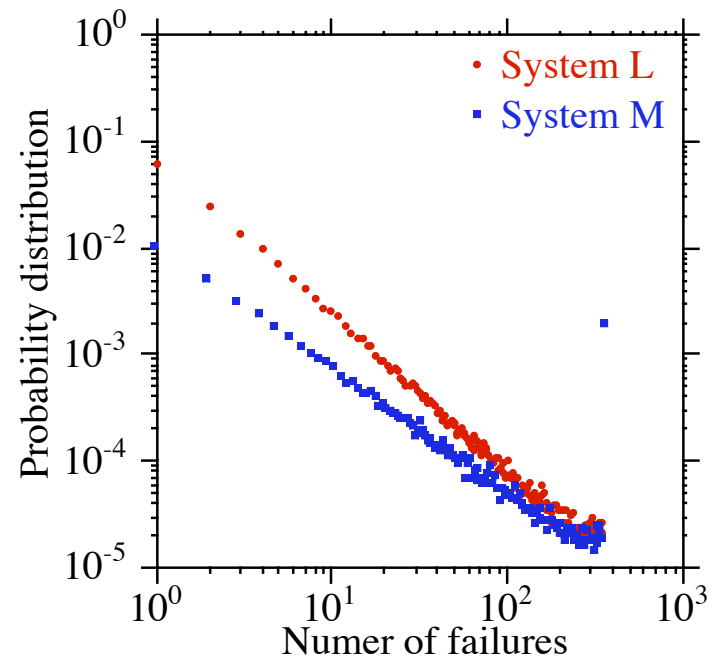

Figure 6: Probability of cascade events of the system size as a function of $\lambda$

In Figures 7 and 8 we see the evolution of a cascade for a case in which there would have been no cascade in $M$ and the cascade in $L$ would have stopped after 4 iterations had the systems been uncoupled. Figure 7 shows the number of failures per iteration as the cascade evolves and in this case the two systems are tightly coupled so number of failures per iteration is approximately the same for both systems.

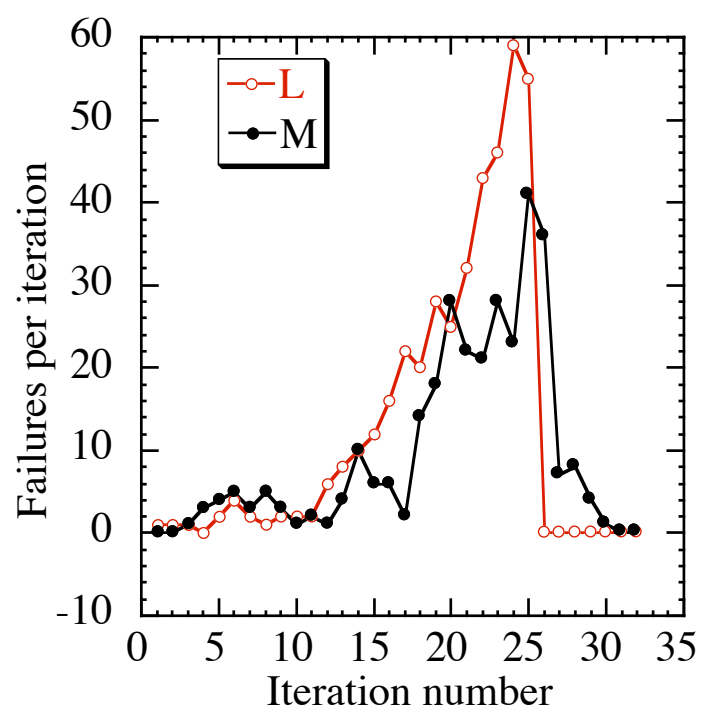

Figure 7: The evolution of failures in a cascade as a function of iteration for both systems.

In figure 8 , which shows the cumulative number of failures in each of the two systems, the cascade can be seen to go all the way to the system size (400) in system $L$ at approximately iteration 25. The cascade stops in system $M$ when it reaches the full system size in $L$ because it is no longer being driven by anything. System $L$ is gone!

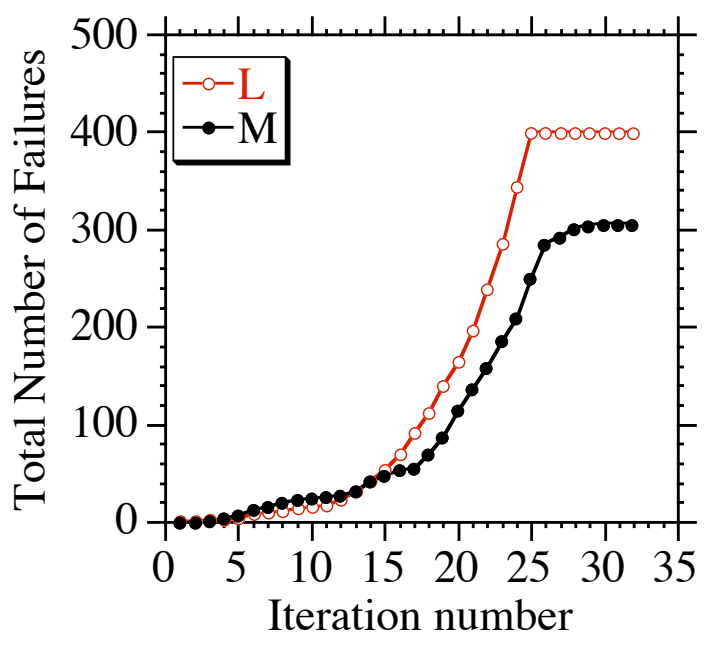

\section{Figure 8: Evolution of the cumulative number of failures in a cascade as a function of iteration for both systems.}

If one thinks of system $L$ as a power transmission system and system $M$ as an information communications system the meaning and effect of the coupling is fairly clear. The two systems are coupled in both directions at the simplest level because the communications system uses power to operate and because the communications system carries the information needed to operate the power transmission system. Failure in one increases the probability of failure in the other. For example a power failure increases the probability of a router failing, leading to information packet losses. This failure in the second system then can react back on the first system increasing its probability of further failure. For example, lack of knowledge of the operating state of a line increases the probability of an overload condition. This process facilitates the propagation of the cascade that is the mechanism by which the critical point is lowered.

Both the numeric and analytic approaches to understanding this model can be extended to cases that relax some of the simplifications we have made. Of most interest is relaxing the symmetry assumption in the coupling. This work will be presented in a subsequent paper. 


\section{A coupled complex system model}

\subsection{The simple dynamical complex system model}

Probabilistic models such as the CASCADE model can shed light on the changes in the critical point and pdf of failures. However, their value is limited by their probabilistic nature. In order to develop sufficient statistics for these measures many realizations with independent initial conditions are performed with no knowledge of earlier cases. We know however that the real systems are deterministic and its state today knows about its state yesterday at least to some degree. Therefore, to investigate the dynamics of these systems we utilize a coupled dynamic complex system model (DCSM).

This DCSM is a cellular automata based model. It is set on a regular grid with fixed interaction rules. The systems we will discuss here are a subset in which the rules are local and the grid is regular. Both of these restrictions are straightforward to generalize (and for some systems other choices make more sense) but we use them as a reasonable starting point.

The rules for the single, uncoupled systems are simple:

1) A node has a certain (usually small) probability of failure $\left(p_{f}\right)$

2) A node neighboring a failed node has another (higher) probability of failing $\left(\mathrm{p}_{\mathrm{s}}\right)$

3) A failed node has a certain (usually high) probability of being repaired $\left(\mathrm{p}_{\mathrm{r}}\right)$

The steps taken in the evolution are equally simple:

At step $\mathrm{t}$

1) The nodes are evaluated for random failure based on their state at the end of the $\mathrm{t}-1$ step.

2) The nodes are evaluated for repair based on their state at the end of the t- 1 step.

3) The nodes are evaluated for failure due to the state of their neighbors at step $\mathrm{t}-1$.

4) All nodes are advanced to their new state

Outages (failures) in these systems can grow and evolve in nonuniform clusters and display a remarkably rich variety of spatial and temporal complexity. They can grow to all sizes from individual node failures to system size events. The repair rate for nodes is usually slower then the time scale of a cascading failure so repairs to an evolving cascade are unlikely. The main difference between this model and the CASCADE model discussed in Section 2, is the continued evolution of the system after a failure. In this system, the "memory" of previous failures is in the structure of failed and fixed nodes in the system. The characteristic time scales of the system are also captured in the repair time and random failure probability. This type of model gives power law tails in the pdf, as before, in addition to long time correlations and anti-correlations between the failures (Figure 9), something that comes from the dynamical memory of the system.

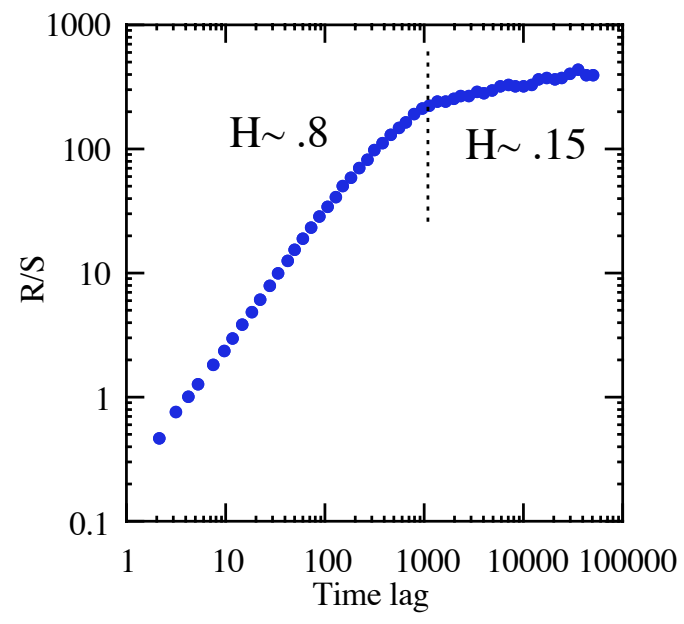

Figure 9: R/S as a function of time lag for a DCSM time series showing a Hurst exponent greater then 0.5 in the mesoscale region, signifying long time correlations.

\subsection{The coupled complex system model}

The coupling of these systems is achieved along similar line to that done in the CASCADE model. Namely, failures in one system change the probability of failure in the other system. The difference being that since, beyond mean field theory, the details of which will be presented elsewhere, we are unable to make much analytic progress with this model we do not worry about simplifying assumptions. Therefore we couple the two dynamical complex systems models DCSM1 and DCSM2 using two coupling variables. The first of these variables is the spatial structure of the coupling. Since all nodes in one system do not need to be coupled to all nodes in the other systems (in fact usually would not be), we can change the fraction of the nodes coupled (randomly or with a fixed structure). See figure 1 for a cartoon representation of this. The second variable is the strength and direction of the coupling. The strength of the coupling is the cross system probability of failure, similar to the $\mathrm{p}_{\mathrm{ML}}$ from the coupled CASCADE model. However we do not restrict this coupling to being symmetric. In reality, some systems failures can have a major impact on its counterpart system while a failure in the counterpart system would have little or no effect on the first system. An example of this might be a co-located pipeline/communications system. The communication system is used to monitor the pipeline state. Failure of the communications system can (or often will) cause a failure (or shutdown) of the pipeline system. The converse is usually not true, a failure in the pipeline, unless it is a catastrophic failure, will have no impact on the communications system. Therefore both the strength and direction can be varied.

\subsection{Preliminary results from the Coupled DCSM}

As described here, the DCSM dynamically arranges itself to sit right at, or near the critical point for a wide range of parameters as long as we are above the percolation limit, which 
will be discussed below. This is why it is called a selforganized critical system. So unlike the CASCADE model we cannot do a simple $\lambda$ scan in DCSM to explore the critical point because the system tries to arrange itself arranges to live at that point. However by changing the parameters in both the local coupling and the cross system coupling we can see changes in the failures which can be made explore similar dynamic changes as the lambda scans in CASCADE.

Figure 10 shows the time series of failures for a coupled system and an uncoupled system (with the same parameters other then the coupling), showing a large change in the dynamics of the system.

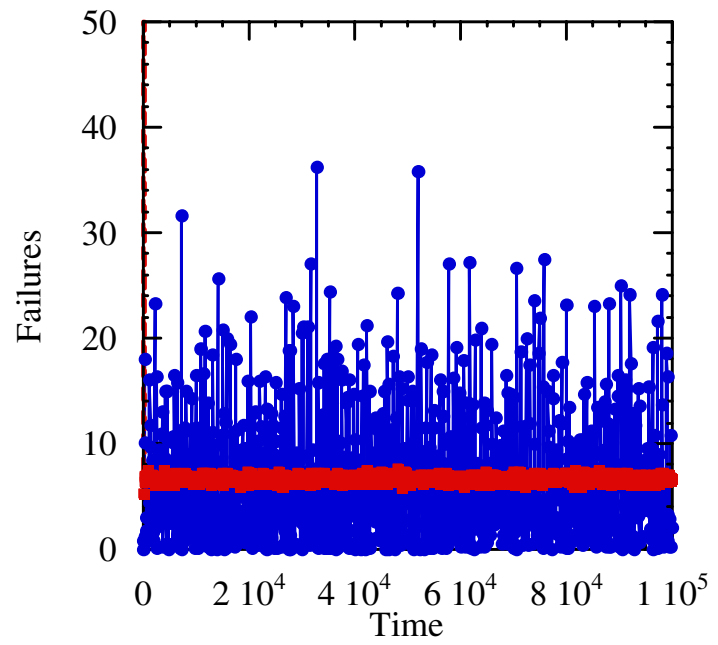

Figure 10: Time series of failure sizes in coupled and
uncoupled DCSM

This figure simply illustrates the extreme differences that can be found between coupled and uncoupled systems, in this case when the coupling is strong and 2 way, causing constant small failures in the 2 systems. To begin a systematic understanding of the parameter space we first note a few of the characteristics of the uncoupled system. First is the local coupling parameter $\mathrm{p}_{\mathrm{s}}$, which when below a certain value makes the system sub-critical to the percolation threshold. This means that when the individual elements are coupled to few other elements, or when the coupling is very weak, the cascading failures will be self-limiting. That is, they will have a very low probability of propagating across the entire system and the distribution (PDF) of failure sizes will be exponential (Fig. 11). The threshold is reached when there is at least one failure on average caused by a failed site. This "percolation" threshold can be analytically approximated [12], using mean field theory, as $\mathrm{P}_{\text {ncrit }} \sim 1 / \mathrm{f}$, with $\mathrm{f}$ being the average number of unfailed sites a site is connected to. This is approximately the number of connections-1 since, during a cascading failure, one of the connections will already be failed. Therefore, for our uncoupled DCSM model with four connections per site, the critical $\mathrm{P}_{\mathrm{n}}$ is about 0.333 . In reality, mean field theory underestimates the threshold value because long time correlations are not considered but the value is not far from that found as seen in figure 12 .

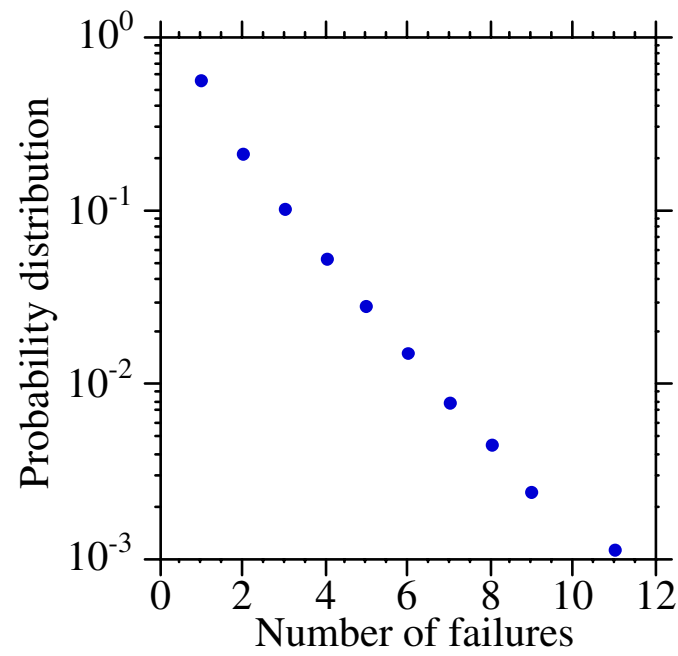

Figure 11: PDF of failure sizes in uncoupled DCSM with coupling parameter $P_{n}=0.1$, significantly less then the critical value. The PDF shows an exponential size distribution.

In this figure the critical point can be characterized as the point at which the average number of new failures caused by a failure $(\lambda)$ equals, or exceeds, one. This is found to be approximately 0.4 for the full DCSM model, just a little above the mean field approximation. Once the system is above the critical point it display all the characteristics of a self-organized complex system.

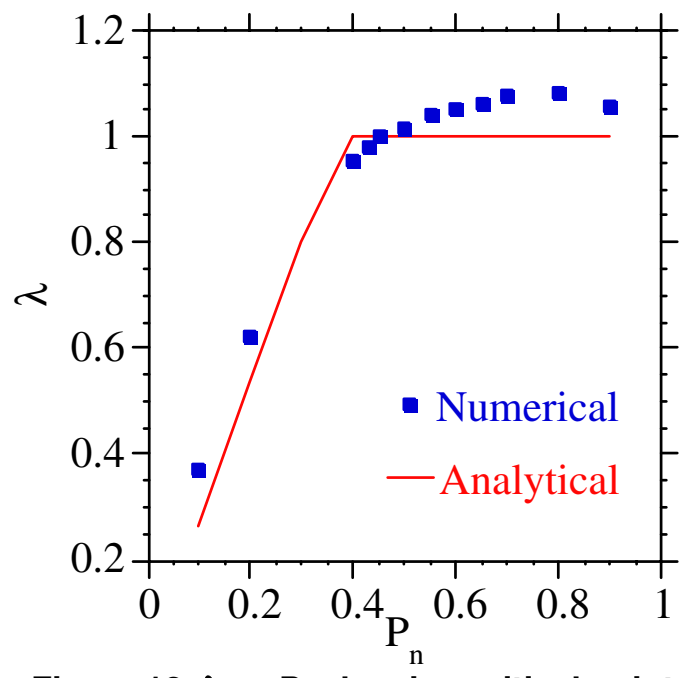

Figure 12: $\lambda$ vs $P_{n}$ showing critical point

These include the long time correlations (Fig. 9) and power law PDFs. The appearance of the power law size distribution as we cross the critical point is shown in figure 13 which has PDFs for a just barely critical case and a case with $\mathrm{P}_{n}$ well above the critical point. The power laws found have exponents of approximately -1 and exhibit the standard exponential cutoff at largest sizes due to finite system size effects. It should be noted that the power law of -1 is in contrast to the CASCADE model which, in the uncoupled case, has a power law of approximately -1.5 and is due to the dynamical evolution of the system. 


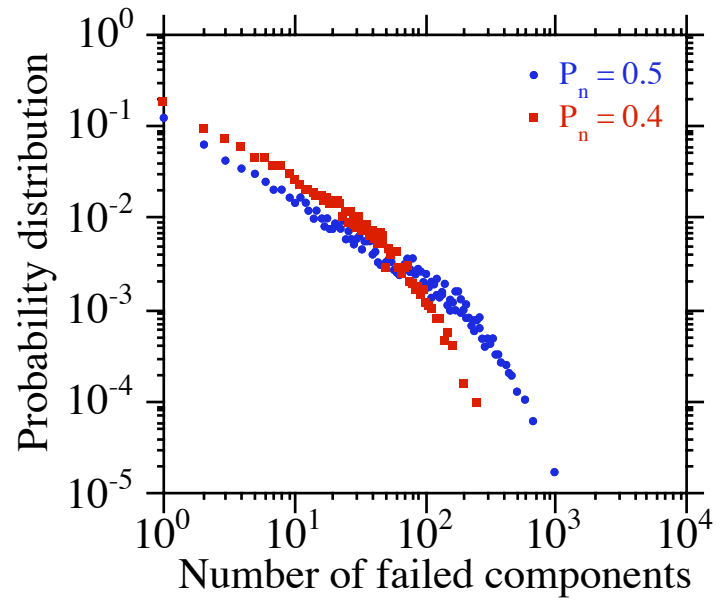

Figure 13: PDFs of failure sizes in 2 uncoupled DCSM calculations with the neighbor coupling parameter $P_{n}=0.4$ and 0.5 , just at and above the critical value. The PDFs show a power law size distribution.

One of the simplest consequences of coupling the 2 systems is to give another propagation path for failures. If this did in fact occur one would expect that the critical point could be crossed by increasing the cross system coupling as well as by increasing the nearest neighbor coupling in a given system. This consequence can be seen in figure 14 in which the $\mathrm{P}_{\mathrm{n}}$ is sub critical but the cross system coupling is able to make the system critical.

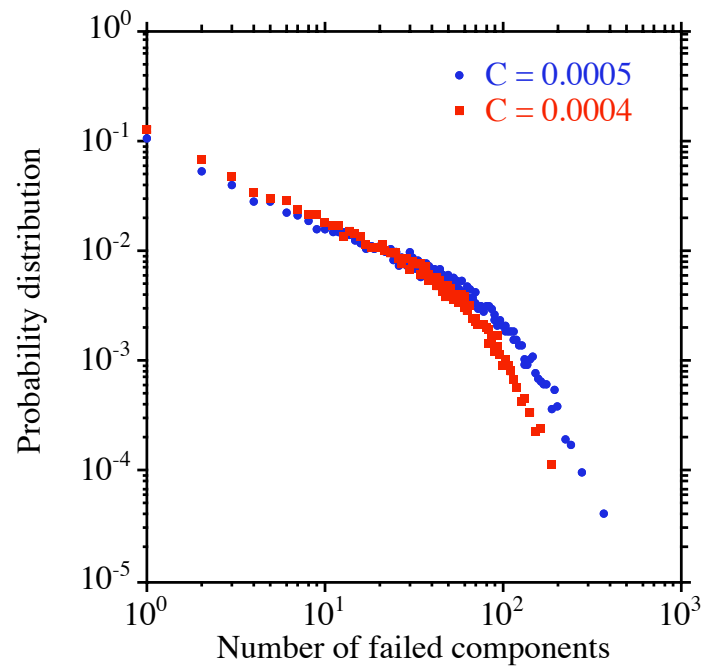

Figure 14: PDFs of failure sizes in 2 coupled DCSM calculations with coupling parameter $P_{n}=0.4$ and 0.5 , just at and above the critical value.

In the coupled case, the power law found is somewhat weaker then the -1 found for the uncoupled system and is approximately 0.8 . The direction of change (ie the weaker power law) is consistent with the effect seen in the coupled CASCADE model discussed in section 2.3, though the coupled DCSM power law is still significantly less steep then the coupled CASCADE result. The actual slope is critical for calculating and understanding the risk of events of various sizes and while changing from an exponential distribution to a power law is much more significant, going from a power law of -1.5 to -.8 will have a large impact on the probability of the largest failures.

Another obvious potential impact of the coupling is the possible synchronization of the failures in the two systems. Using a measure developed by Gann et al in [13] for synchronization, we investigate this effect. Figure 15 shows the synchronization function described in [13] which is basically an average normalized difference between events in the 2 systems. For this measure, a value of 1 means the difference is effectively $100 \%$ or no synchronization, while a value of 0 means all events are the same in the 2 systems, or they are synchronized. These values are then plotted as a function of the event sizes. It can be readily seen that small events for all three of the coupling strengths are largely uncorrelated (unsynchronized). The synchronization however increases as the size increases. This makes physical sense since as the even gets larger there are more sites interacting and this increases the probability that a failure in one system will trigger a failure in the other system. It should be noted that this is likely to be sensitive to the spatial homogeneity of the coupling that is being investigated.

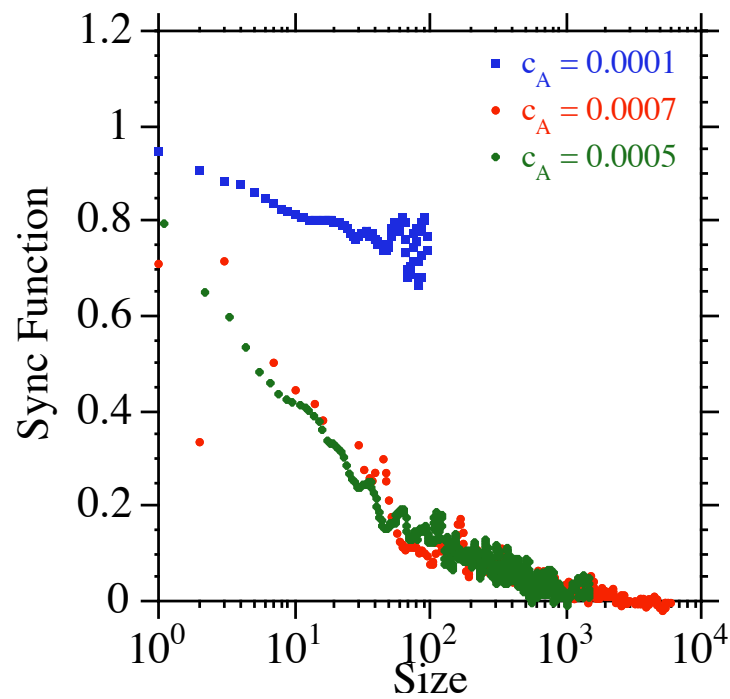

Figure 15: Synchronization functions for coupled DCSM calculations for 3 values of the coupling parameter $C_{a}$. A value of 1 is unsynchronized and 0 is synchronized.

This synchronization of large events is important in assessing the impact of the coupling. It may be that small failures in one system are unlikely to trigger a failure in the coupled system, however if a large failure is likely to trigger a coupled failure then the dynamical state of system one (ie it's proximity to a major failure) becomes very critical in assessing the risk of failure of the perhaps more reliable system two.

The results presented here have been for a very small subset of the parameter space. That subset being, symmetric homogeneous coupling with an increased failure probability from an coupled failed or failing site. The rest of the parameter space described earlier is being investigated and will be reported on later. 


\section{Discussion and Conclusions}

Modern societies rely on the smooth operation of many of the infrastructure systems. We normally take them for granted. However, we are typically shocked when one of these systems fails. Therefore, understanding these systems is a high priority for ensuring security and social wellbeing. Because none of these infrastructure systems operate in a vacuum, understanding how these complex systems interact with each other gains importance when we recognize how tightly coupled some of these systems are. Because of the great complexity of even the individual systems it is unrealistic to think that we can presently dynamically model interacting infrastructure systems in full detail.

In this paper, we have investigated some of the general features of interactions between infrastructure system by using very simple models. We look for general dynamical features without trying to capture the details of the individual systems. From this we try to build a hierarchy of models with increasing levels of detail for these systems.

Here, we have shown two such models. One is a probabilistic model, CASCADE. The other model is a dynamic complex system model (DCSM) which can work in a selforganized critical state. Both models are characterize by a percolation threshold above which cascading failures of all sizes are possible, In both models this threshold can be characterized by the branching parameter $\lambda$, the average number of new failures caused by a failure. The percolation point is at $\lambda=1$, where the probability density of failures for CASCADE is a power law with exponent -1.5 while for DCSM it is somewhat closer to -1.0 . These exponents are close to the one found in analysis of blackout data.

It has been found that symmetric coupling of these systems actually decreases the threshold. That is, it makes access to the critical point easier, which means that the systems when coupled are more susceptible to large-scale failures and a failure in one system can cause a similar failure in the coupled system. The parameter $\lambda$, can be also used to characterize the cascading threshold in the coupled systems. This suggests the existence of a metric that can be generalized for practical application to more realistic systems.

For the DCSM model in addition, it is found that large failures are more likely to be "synchronized" across the two dynamical systems, which is likely to be the reason that the power law found in the probability of failure with size is less steep with the coupling. This means that in the coupled systems there greater probability of large failures and less of smaller failures.

With the DCSM model other important aspects of the infrastructure can be explored, such as non-uniform and nonsymmetric couplings. This will be the object of future studies.

With this model there is a large parameter space that must be explored with different regions of parameter space having relevance to different infrastructure systems. There is also a rich variety of dynamics to be characterized. Characterizing the dynamics in the different regimes is more then an academic exercise since as we engineer higher tolerances in individual systems and make the interdependencies between systems stronger we will be exploring these new parameter regimes the hard way, by trial and error. Unfortunately error in this case has the potential to lead to global system failure. By investigating these systems from this high level, regimes to be avoided can be identified and mechanisms for avoiding them can be explored.

\section{Acknowledgments}

Ian Dobson and David Newman gratefully acknowledge support in part from NSF grants ECS-0216053 and ECS-0214369. Ian Dobson and B. A. Carreras gratefully acknowledge coordination of part of this work by the Consortium for Electric Reliability Technology Solutions and funding in part by the Assistant Secretary for Energy Efficiency and Renewable Energy, Office of Power Technologies, Transmission Reliability Program of the U.S. Department of Energy under contract 9908935 and Interagency Agreement DE-A1099EE35075 with the National Science Foundation. Part of this research has been carried out at Oak Ridge National Laboratory, managed by UT-Battelle, LLC, for the U.S. Department of Energy under contract number DE-AC05$00 \mathrm{OR} 22725$.

\section{References}

[1] Richard G. Little, Toward More Robust Infrastructure: Observations on Improving the Resilience and Reliability of Critical Systems, in Proceedings of the 36th Annual Hawaii International Conference on System Sciences (HICSS'03).

[2] S. M. Rinaldi, Modeling and Simulating Critical Infrastructures and Their Interdependencies, in Proceedings of the 37th Annual Hawaii International Conference on System Sciences (HICSS'04), (Big Island, HI, USA), IEEE Computer Society Press, Jan. 2004.

[3] S. M. Rinaldi, J. P. Peerenboom, and T. K. Kelly Identifying, understanding, and analyzing critical infrastructure interdependencies, IEEE Control Systems Magazine, p. 11, December 2001.

[4] I. Dobson, B. A. Carreras, and D. E. Newman, A probabilistic loading-dependent model of cascading failure and possible implications for blackouts, 36th Hawaii International Conference on System Sciences, Maui, Hawaii, Jan. 2003.

[5] I. Dobson, B.A. Carreras, D.E. Newman, Probabilistic loaddependent cascading failure with limited component interactions, IEEE International Symposium on Circuits and System, Vancouver Canada, May 2004.

[6] I. Dobson, B. A. Carreras, and D. E. Newman, A loadingdependent model of probabilistic cascading failure, to appear in Probability in the Engineering and Informational Sciences, 2005.

[7] B.A. Carreras, V.E. Lynch, I. Dobson, D.E. Newman, Critical points and transitions in an electric power transmission model for cascading failure blackouts, Chaos, vol. 12, no. 4, December 2002, pp. 985-994.

[6] I. Dobson, J. Chen, J.S. Thorp, B. A. Carreras, and D. E. Newman, Examining criticality of blackouts in power system models with cascading events, 35th Hawaii International Conference on System Sciences, Hawaii, Hawaii, Jan. 2002.

[9] Charles Perrow, Normal accidents, Princeton University Press, 1984. 
[10] I. Dobson, B.A. Carreras, D.E. Newman, A branching process approximation to cascading load-dependent system failure. 37th

Hawaii International Conference on System Sciences, Hawaii, January 2004.

[11] T.E. Harris, Theory of branching processes, Dover NY 1989.

[12] B. Drossel and F. Schwabl, Physica A 199, 183 (1993).

[13] R. Gann et al Phys. Rev. E 69, 046116 (2004). 